\title{
TEMA-2017: Acromegalia
}
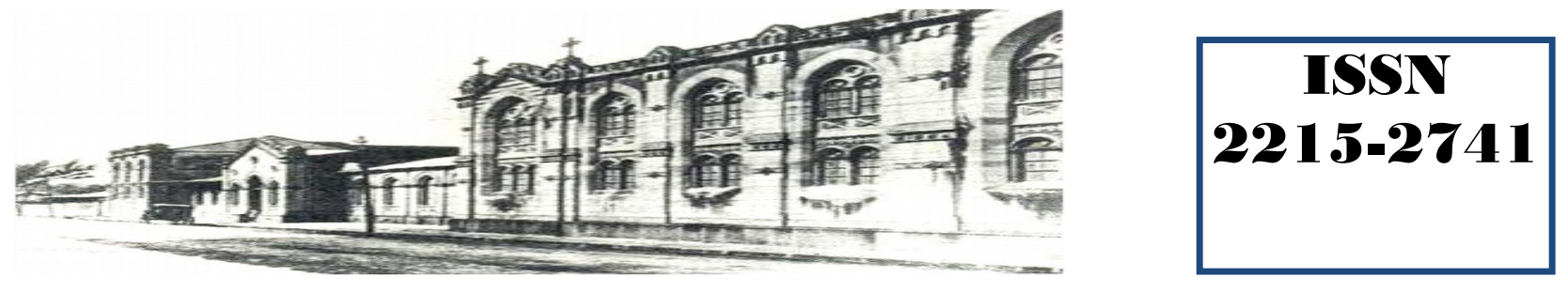

Haspital San quan de Dios. San José. Casta Rica. Fundado en 1845

Recibido:

Aceptado:
$12 / 03 / 2017$

$15 / 04 / 2017$

\author{
Gloriana Herra Leandro ${ }^{1}$ \\ Karolina Rojas Núñez ${ }^{2}$ \\ Marcela Alfaro Rodríguez ${ }^{3}$
}

${ }^{1}$ Médico General. Universidad de Costa Rica. Correo electrónico: draherra23@gmail.com

${ }^{2}$ Médico General. Universidad de Costa Rica. Correo electrónico: dra.karojas@gmail.com

${ }^{3}$ Médico especialista en Endocrinología. Universidad de Costa Rica. Correo electrónico:

\section{RESUMEN}

La acromegalia es una enfermedad causada por la producción excesiva de hormona de crecimiento, usualmente consecuencia de un adenoma hipofisario. Los adenomas presentan dos subtipos histológicos, densamente granulados o escasamente granulados. Las manifestaciones clínicas incluyen visceromegalia, cambios en el tejido blando, sudoración excesiva, efectos de masa por el tumor, trastornos cardiovasculares, metabólicos, respiratorios, manifestaciones en huesos y articulaciones o secuelas endocrinas. Para el diagnóstico, es importante la medición del factor de crecimiento insulínico tipo $1 \mathrm{y}$ un test de tolerancia oral a la glucosa para determinar el nivel de hormona de crecimiento. Existen diversas modalidades de tratamiento, tales como quirúrgico, médico o radioterapia. El tratamiento quirúrgico es el de primera línea en la mayoría de pacientes con acromegalia, sin embargo la elección de la intervención se basa en múltiples factores, se debe individualizar el tratamiento y brindar valoración por parte de un equipo multidisciplinario.
En ocasiones se requiere más de una modalidad para alcanzar las metas del tratamiento.

\section{PALABRAS CLAVE}

Acromegalia. Adenoma hipofisario. Hormona de crecimiento. Factor de crecimiento insulínico tipo 1. Análogos de somatostatina. Agonistas de dopamina. Antagonista del receptor de hormona de crecimiento.

\section{ABSTRACT}

Acromegaly is a disease caused by the excessive production of growth hormone, commonly by a pituitary adenoma. There are two histologic subtypes, densely granulated or sparsely granulated. The clinical manifestations include visceromegaly, changes in soft tissue, excessive sweating, tumor mass effects, cardiovascular, metabolic and respiratory disorders, manifestations in bones and joints or endocrine repercus- 
sions. To make the diagnosis, it is important to measure insulin-like growth factor-1 levels and an oral glucose tolerance test to determine growth hormone levels. The different modalities of treatment include surgery, medical therapy or radiotherapy. Surgery is the first line treatment for most of the patients with acromegaly, although the decision is based in multiple factors, the treatment should be individualized and the patient should be evaluated by a multidisciplinary team. Some cases may need combination therapy to achieve the treatment goals.

\section{KEY WORDS}

Acromegaly. Pituitary adenoma. Growth hormone. Insulin-like growth factor-1. Somatostatin analogues. Dopamine agonists. Growth hormone receptor antagonist.

\section{INTRODUCCIÓN}

El término "acromegalia" fue acuñado por primera vez en 1886 por el neurólogo francés Pierre Marie, asociado a una enfermedad específica con una clínica característica. ${ }^{1}$ La acromegalia y el gigantismo se deben a la producción excesiva de hormona de crecimiento $(\mathrm{GH})$, generalmente por un adenoma hipofisario. ${ }^{2}$

La principal diferencia entre ambas condiciones es que el gigantismo resulta de la producción excesiva de $\mathrm{GH}$ durante el período de crecimiento activo, es decir, se origina durante la infancia o la adolescencia, mientras que la fisis o cartílago de crecimiento permanece abierto; en cambio la acromegalia resulta de exceso de GH que se produce después de que se ha dado el cierre de la fisis o fusión epifisaria. ${ }^{3}$

Aunque la GH puede tener efectos directos en los tejidos periféricos, la mayoría de los efectos que promueven el crecimiento son mediados por el factor de crecimiento insulínico tipo 1 (IGF-1). ${ }^{2}$ El diagnóstico de acromegalia es antecedido por alrededor de 10 años de enfermedad activa. ${ }^{4,5} \mathrm{La}$ acromegalia presenta una prevalencia de 36-39 casos por millón y una incidencia de 3-4 casos por millón por año. ${ }^{6}$ En cambio, los casos de gigantismo son extremadamente raros. ${ }^{3}$
Los adenomas somatotropos son de origen monoclonal. ${ }^{4}$ Entre los genes involucrados en dicha expansión clonal somatotropa, estudiados en modelos animales, se encuentran: el gen supresor del retinoblastoma, p27, gen MENIN, mutaciones en Ras, mutaciones en el gen transformante de tumores de pituitaria y mutaciones en el gen supresor de tumores o proteína que interacciona con el receptor arylhydrocarbon (AIP). ${ }^{7}$ Los adenomas pituitarios secretores de GH se clasifican en dos subtipos histológicos según el patrón de citoqueratina citoplasmática. Existen los adenomas densamente granulados, los cuales crecen lentamente y se manifiestan en personas mayores de 50 años. Y los adenomas escasamente granulados, estos crecen más rápidamente, son más comunes en personas jóvenes, sugieren lesiones más invasivas y tienen menor respuesta a la terapia con análogos de somatostatina.,

\section{DISCUSIÓN}

\section{Manifestaciones Clínicas}

Las manifestaciones clínicas dependen de las concentraciones de GH e IGF-1, la edad del paciente, el tamaño del tumor y el tiempo de retraso en el diagnóstico. ${ }^{4}$ Ante la sospecha de acromegalia, se debe consultar y examinar a los pacientes en la búsqueda de signos y síntomas típicos de dicha patología. Entre las complicaciones asociadas con la hipersecreción de GH e IGF-1 se incluyen: visceromegalia, cambios en el tejido blando, sudoración excesiva y crecimiento mandibular. ${ }^{6,8}$

También pueden ocurrir efectos de masa por el tumor, los cuales incluyen cefalea, defectos en el campo visual, hiperprolactinemia, sección del tallo hipofisario e hipopituitarismo (que puede incluir hipotiroidismo, hipogonadismo e hipocortisolismo). En cuanto a los factores cardiovasculares, la acromegalia se puede asociar con hipertrofia (biventricular o septal asimétrica), insuficiencia cardíaca congestiva, enfermedad coronaria, arritmias, hipertensión y cardiomiopatía. Además, se relaciona con factores metabólicos, entre ellos intolerancia a los carbohidratos, diabetes mellitus y resistencia a la insulina. 
La acromegalia puede presentar manifestaciones respiratorias debido a macroglosia, defecto mandibular, obstrucción de la vía aérea superior, trastornos del sueño, síndrome de apnea del sueño (ya sea por origen central u obstructivo) o disfunción ventilatoria. Por otra parte, se pueden presentar manifestaciones en huesos y articulaciones, tales como aumento del grosor del cartílago articular, artralgias y artritis, síndrome del túnel carpal u osteopenia. La acromegalia puede llevar a secuelas endocrinas como bocio, hipercalciuria, galactorrea, disminución de la libido o disfunción sexual, así como irregularidades menstruales en las mujeres. ${ }^{5}$

La acromegalia se asocia con aumento de los pólipos en colon y se puede asociar con incremento del riesgo de cáncer colorrectal, pero no con mortalidad por cáncer. Se recomienda una colonoscopia de tamizaje al momento del diagnóstico en adultos. Si la colonoscopia es negativa, se recomienda el tamizaje igual a la población general. Si los valores de IGF-1 persisten elevados, el tamizaje debe ser más frecuente. Si la colonoscopia resulta anormal, el seguimiento debe ser según las guías clínicas. ${ }^{9}$

Es fundamental sospechar el diagnóstico y brindar el tratamiento oportuno. Sin tratamiento, la exposición prolongada a hipersecreción de $\mathrm{GH}$ e IGF-1 se puede asociar con aumento en el riesgo de morbilidad y mortalidad, deterioro de comorbilidades y pobre calidad de vida. Asimismo, algunos estudios sugieren que se puede asociar con peor perfil psicosocial. ${ }^{6}$

\section{Diagnóstico}

En las etapas tempranas de la acromegalia, puede que no se manifieste con características claras para realizar el diagnóstico. Se debe sospechar esta patología en las personas que presenten dos o más de las siguientes comorbilidades: diabetes de nueva aparición, artralgias difusas, hipertensión de nueva aparición o de difícil manejo, enfermedad cardíaca como hipertrofia biventricular y disfunción diastólica o sistólica, fatiga, cefaleas, síndrome del túnel carpal, síndrome de apnea del sueño, diaforesis, pérdida de visión, pólipos en colon y maloclusión progresiva. ${ }^{8}$
Se recomienda la medición de los niveles de IGF-1 en los contextos que se mencionan a continuación:

a) Pacientes con manifestaciones clínicas típicas de acromegalia, especialmente aquellas acrales y faciales.

b) Pacientes sin manifestaciones típicas de acromegalia pero con algunas condiciones asociadas a esta patología (como síndrome de apnea del sueño, diabetes mellitus tipo 2, artritis debilitante, síndrome del túnel carpal, hiperhidrosis o hipertensión).

c) También es adecuado medir IGF-1 sérica para descartar acromegalia en pacientes con una masa pituitaria. $^{10}$

Es importante al analizar el resultado del nivel de IGF-1 sérico, correlacionarlo con aquellos de sujetos de la misma edad y género. En general, la medición de un valor al azar de IGF-1 (considerado un marcador de la secreción integrada de $\mathrm{GH}$ ) es adecuada para diagnóstico y para monitoreo después de una intervención terapéutica. ${ }^{8} \mathrm{La}$ medición del IGF-1 es la mejor prueba para el tamizaje de la acromegalia, debido a que (en comparación con la $\mathrm{GH}$ sérica) tiene una menor variabilidad a lo largo del día, mayores concentraciones séricas, una vida media sérica más larga y tiene una alta sensibilidad.

Por otra parte, la GH sérica tiene amplios rangos de variabilidad normal a lo largo del día, una vida media corta y una gran variación de su secreción ante la presencia de distintos estímulos tales como la comida, el ejercicio y el estrés, y ante factores como el peso corporal, la edad, el estado nutricional y los períodos de sueño. En general, para confirmar el diagnóstico de acromegalia se requiere de una prueba confirmatoria, y la prueba de tolerancia a la glucosa oral es la prueba de mayor especificidad para la confirmación del diagnóstico del exceso de secreción de GH. ${ }^{11}$

Se considera normal un valor de $\mathrm{GH}$ menor de $1 \mathrm{ng} / \mathrm{mL}$ tras un test de tolerancia oral a la glucosa, el cual consiste en $75 \mathrm{~g}$ de glucosa por vía oral, seguido de mediciones de GH cada 30 minutos durante 120 minutos. ${ }^{8}$ Se ha descrito también un procedimiento con una determina- 
ción única de $\mathrm{GH}$ a los 120 minutos que podría ser tan útil como la curva completa. ${ }^{12}$ En aquellos sujetos con niveles séricos de IGF-1 elevados o equívocos, se recomienda confirmación del diagnóstico demostrando falta de supresión de la $\mathrm{GH}$ a menos de $1 \mathrm{ng} / \mathrm{mL}$ tras hiperglicemia inducida con la carga oral de glucosa. ${ }^{10}$ Es decir, el diagnóstico se realiza cuando al menos una de las mediciones de la GH sérica se encuentra mayor de $1 \mathrm{ng} / \mathrm{mL} .^{11}$

Cierta literatura, incluyendo las guías de acromegalia de la AACE, sugieren que el nadir de hormona de crecimiento sérica tras la administración de glucosa se reduzca a $0.4 \mathrm{ng} / \mathrm{mL}$ con el fin de aumentar la sensibilidad de la prueba. ${ }^{8}$

Además del diagnóstico bioquímico de acromegalia, se recomienda realizar un estudio de imagen que permita visualizar el tamaño y la apariencia del tumor, así como la presencia y grado de extensión paraselar. La técnica de elección es la resonancia magnética ( $\sin$ y con medio de contraste), y en caso de que esté containdicada o no disponible, se sugiere tomografía computarizada. En caso de compresión del quiasma óptico en el estudio de imagen o si el paciente aqueja disminución de la visión periférica, es prudente realizar estudios del campo visual. ${ }^{8,10}$

Asimismo, se deben extender los estudios bioquímicos para evaluación de la función hipofisaria anterior y posterior (por un potencial hipopituitarismo) y con el nivel de prolactina sérica (para evaluar presencia de hiperprolactinemia). ${ }^{8}$ La hiperprolactinemia con o sin galactorrea se puede desarrollar aproximadamente en un $30 \%$ de pacientes, principalmente debido a compresión del tallo pituitario o en ocasiones debido a la secreción mixta de $\mathrm{GH}$ y prolactina proveniente del tumor. ${ }^{5}$

\section{Tratamiento}

Las metas del tratamiento de la acromegalia incluyen: control de los síntomas (mejorando a la vez la calidad de vida), control del crecimiento tumoral con preservación de la función hipofisaria, normalizar las concentraciones bioquímicas de GH e IGF-1, y revertir el exceso de morbilidad y mortalidad asociados con esta patología. $^{4,7}$
Existen diversas modalidades disponibles, tales como tratamiento quirúrgico, tratamiento médico o radioterapia. ${ }^{4,7,13}$ La elección de la intervención se basa en múltiples factores, se debe individualizar el tratamiento y brindar valoración por parte de un equipo multidisciplinario. En ocasiones se requiere más de una modalidad para alcanzar las metas del tratamiento. ${ }^{7}$

\section{Tratamiento Quirúrgico}

Es el tratamiento de primera línea en la mayoría de pacientes con acromegalia. ${ }^{4,7,8,10,13,14}$ La técnica más recomendada es la transesfenoidal. ${ }^{7,10,13,14}$ Las vías para extirpar el tumor pueden ser translabial o transnasal. ${ }^{15} \mathrm{La}$ cirugía transesfenoidal transnasal endoscópica ofrece las siguientes ventajas: mejor visualización, preservación de la función sinonasal, mejora la resección de los componentes supraselar y paraselar de los adenomas y reducción de la estadía hospitalaria comparado con las técnicas transesfenoidal translabial o transcraneal. Asimismo, al mejorar la visualización e identificación de componentes extraselares, mejora la extensión de la resección y la seguridad. Las complicaciones de dicha técnica son usualmente menores e incluyen diabetes insípida transitoria y fuga de líquido cefalorraquídeo. ${ }^{14}$ En los casos de tumores invasivos con un marcado crecimiento supraselar, puede ser necesario el acceso transcraneal. ${ }^{15}$

Se ha sugerido un posible rol de la terapia médica con análogos de somatostatina preoperatoria para reducir el riesgo quirúrgico y para mejorar el resultado a nivel bioquímico, sin embargo se requieren estudios a futuro para apoyar su uso de forma generalizada. ${ }^{8}$ Las guías de la Endocrine Society se oponen al uso de rutina de terapia médica prequirúrgica para mejorar el control bioquímico postquirúrgico. Por otra parte, sí sugieren el uso de análogos de somatostatina preoperatoria para reducir el riesgo quirúrgico en pacientes con edema faríngeo severo y apnea del sueño o con insuficiencia cardíaca de alto gasto. ${ }^{10}$

Es fundamental la monitorización bioquímica de los niveles de GH y de IGF-1 después de la cirugía para un adecuado manejo y para guiar el tratamiento adicional en caso de ser necesario. ${ }^{6} \mathrm{Se}$ define control óptimo de la enfermedad con una medición al azar de la GH menor a $1 \mathrm{ng} / \mathrm{mL} .{ }^{16}$ 
Además, los niveles séricos de IGF-1 se deben medir a las 12 semanas posterior a la cirugía y deben estar en el rango normal ajustado a la edad; se puede considerar otra medición en otras 9-12 semanas ya que la normalización en los niveles de IGF-1 puede retrasarse. ${ }^{6,16}$ La medición de los niveles de la $\mathrm{GH}$ tras un test de tolerancia oral a la glucosa (OGTT) se debe reservar para casos en los cuales exista incertidumbre (si los niveles de IGF-1 permanecen elevados a los 3-6 meses después de la cirugía). ${ }^{16,17}$ Existe evidencia de que un nadir de $\mathrm{GH}$ menor a $0.4 \mathrm{ng} / \mathrm{mL}$ (con ensayos ultrasensibles) puede definir control de la enfermedad. ${ }^{6,8,16}$

Se sugiere una imagen de resonancia magnética a las 12 semanas posterior a la cirugía para evaluar la posibilidad de tumor residual. ${ }^{8,10}$ Además, se debe valorar la función hipofisaria, incluyendo los ejes tiroideo y gonadal, entre las 6 y 12 semanas posterior a la cirugía. ${ }^{8}$ También es necesario un monitoreo estricto y a largo plazo de la integridad del eje hipotálamo-pituitaria-adrenal (HPA) en todos los pacientes acromegálicos, independientemente del tipo de tratamiento. Esto es importante ya que la función del eje HPA puede empeorar con el tiempo en los pacientes con acromegalia, pueden llegar a desarrollar síntomas y signos de insuficiencia adrenal. ${ }^{18}$

Sin embargo, aproximadamente un $40-60 \%$ de los macroadenomas no se logran controlar sólo con cirugía, principalmente aquellos que ya presentan invasión extraselar. ${ }^{14}$ Pacientes con adenomas de mayor tamaño (más de $20 \mathrm{~mm}$ ) y con un nivel de hormona de crecimiento mayor de 50 $\mathrm{ng} / \mathrm{mL}$ pueden requerir tratamiento médico e incluso en ocasiones terapia con radiación para controlar la hipersecreción de dicha hormona. ${ }^{17}$

\section{Tratamiento Médico}

Esta modalidad se indica en los siguientes casos: pacientes con contraindicación para la cirugía, falla del tratamiento quirúrgico (persistencia de la enfermedad), tumores invasivos (combinación de terapias), espera del efecto de la radioterapia o como se mencionó anteriormente, en ocasiones se sugiere su uso previo a la intervención quirúrgica para mejorar el resultado. ${ }^{15}$
El objetivo del tratamiento médico es reducir el nivel de $\mathrm{GH}$ en ayunas y que las concentraciones de IGF-1 se reduzcan de manera que se acerquen al valor normal. Los estudios se deben actualizar debido a que existen ensayos más sensibles y específicos. Además, el tratamiento médico puede prevenir que el tumor continúe creciendo o producir alivio de los síntomas y signos debidos a la compresión por el efecto de masa. La disminución del tumor puede ocurrir a los 3 meses de haber iniciado esta terapia. ${ }^{19}$

Existen tres formas de tratamiento médico para el manejo de la acromegalia. Dos dirigidas al adenoma hipofisario con mecanismo basado en el receptor, se incluyen los análogos de somatostatina (octreótido, lanreótido y pasireótido) y los agonistas de dopamina (como cabergolina). Uno de ellos es dirigido a disminuir y/o bloquear los efectos de la hormona de crecimiento a nivel periférico (el antagonista del receptor de hormona de crecimiento llamado Pegvisomant). ${ }^{19,20,21}$

\section{Análogos de Somatostatina}

La somatostatina (un péptido hipotalámico) inhibe la secreción de GH por parte de las células somatotrofas hipofisarias, al unirse a los receptores de somatostatina. Existen 5 subtipos conocidos de dichos receptores, el control de la liberación de GH se da vía los subtipos 2 y 5 . Los adenomas somatotrofos se caracterizan por una mayor densidad de ambos subtipos (2 y 5). Sin embargo la expresión de los receptores de somatostatina en el tejido del adenoma es no homogéneo y es específico de cada tumor; por lo que la respuesta a los análogos de somatostatina es variable según el adenoma en particular. ${ }^{20}$

Otro mecanismo de acción de los análogos de somatostatina que se ha discutido es la antiangiogenesis, ya sea de forma directa (por medio de factores de crecimiento) o a través de efectos inmunomoduladores. ${ }^{20}$

Existen formulaciones de liberación prolongada de lanreótido y octreótido, las cuales están dirigidas principalmente al subtipo 2 del receptor de somatostatina y tienen una eficacia similar. ${ }^{8,18}$ Las formulaciones disponibles se mencionan a continuación. Lanreótido de liberación prolongada para inyección subcutánea (depósito o auto- 
gel), cuya dosis inicial es $90 \mathrm{mg}$ al mes, con titulaciones hacia abajo a $60 \mathrm{mg}$ o hacia arriba hasta $120 \mathrm{mg}$ al mes. Esta dosis de $120 \mathrm{mg}$ se puede administrar en intervalos de 8 semanas, dependiendo de la respuesta bioquímica.

Octreótido LAR (de liberación prolongada) se inyecta vía intramuscular, a una dosis inicial de 20 mg al mes, con titulación cada 3 a 6 meses hacia abajo a $10 \mathrm{mg}$ o hacia arriba hasta $40 \mathrm{mg}$ al mes. ${ }^{10}$

Pasireótido es un análogo de somatostatina con una alta afinidad por el subtipo 5 y con un poco de menor afinidad por el subtipo 2 de los receptores de somatostatina. Han surgido datos de resultados de los ensayos clínicos que respaldan la eficacia y seguridad de pasireótido en pacientes con acromegalia. ${ }^{21}$

Los estudios enfocados en la eficacia y seguridad a largo plazo (más de 3 años) de los análogos de somatostatina han tenido resultados reconfortantes. Se puede considerar disminuir la dosis o reducir la frecuencia de administración en pacientes que los necesitan durante un periodo prolongado para el control de la acromegalia; sin embargo se deben evaluar a intervalos regulares para asegurar el mantenimiento del efecto terapéutico. ${ }^{19}$ Entre los efectos adversos se conocen: eritema en el sitio de inyección, náuseas o vómitos, anorexia, malestar abdominal, flatulencia, diarrea, cálculos biliares e intolerancia a la glucosa. $^{4,20}$

Si con el aumento de la dosis no se logra alcanzar las metas bioquímicas, se puede dar terapia combinada. Los análogos de somatostatina se pueden combinar con Pegvisomant para además bloquear el receptor de hormona de crecimiento e inhibir sus efectos. Los análogos de somatostatina también se pueden combinar con agonistas de dopamina y así contribuir a la regulación de la secreción hipofisaria de hormona de crecimiento. ${ }^{8,21}$

\section{Agonistas de Dopamina}

Se encuentran disponibles dos agonistas de dopamina, cabergolina y bromocriptina, sin embargo la cabergolina es más efectiva y mejor tolerada. Se puede considerar su uso particularmente en pacientes con actividad bioquímica leve, como niveles de IGF-1 séricos moderadamente elevados en ausencia o presencia de análogos de somatostatina. ${ }^{8,20}$ No se ha demostrado claramente la relación entre la respuesta de la $\mathrm{GH}$ a la cabergolina con la presencia o ausencia de hiperprolactinemia. ${ }^{6,8}$

La cabergolina se utiliza a dosis variable, se puede iniciar con $1 \mathrm{mg}$ por semana, sin embargo habitualmente se requieren dosis más elevadas, llegando incluso hasta $7 \mathrm{mg}$ por semana para lograr normalizar la alteración bioquímica. ${ }^{4} \mathrm{Se}$ recomienda determinar los niveles de $\mathrm{GH}$, prolactina e IGF-1 a las 4 a 6 semanas posterior al cambio de dosis. ${ }^{6,8}$

Entre los posibles efectos secundarios de los agonistas de dompamina se mencionan los gastrointestinales (diarrea o constipación), hipotensión ortostática, cefalea y congestión nasal. La cabergolina a dosis altas para tratar la Enfermedad de Parkinson, se ha asociado con anormalidades valvulares evidenciadas por ecocardiograma. Sin embargo el efecto clínico de este hallazgo en pacientes con acromegalia no es claro. ${ }^{8,20}$

\section{Antagonista del receptor de hormona} de crecimiento

Pegvisomant es un fármaco diseñado para bloquear al receptor de $\mathrm{GH}$ y por tanto, la acción de dicha hormona. Es un análogo de la GH con una sustitución de un aminoácido en la posición 120 que lo hace antagonista. ${ }^{2,20}$

Para valorar los resultados bioquímicos del tratamiento con Pegvisomant, se utiliza la normalización de los niveles de IGF-1. Los valores de GH no son útiles como marcadores bioquímicos, ya que la secreción endógena de esta hormona puede aumentar durante el tratamiento debido a retroalimentación negativa y particularmente por la reactividad cruzada de la GH con Pegvisomant en la mayoría de ensayos. ${ }^{2,20}$

Muchos estudios han demostrado la normalización o una marcada reducción de IGF-1 en pacientes con acromegalia tratados con Pegvisomant. Este fármaco mejora el síndrome clínico de acromegalia en un alto porcentaje de pacientes, mejora el metabolismo de la glucosa, la cali- 
dad de vida, así como las complicaciones cardiovasculares y esqueléticas. $^{2}$

Pegvisomant es utilizado como tratamiento médico en pacientes con una respuesta inadecuada o no tolerantes a los análogos de somatostatina. En personas con respuesta parcial a los mismos, se puede considerar Pegvisomant en terapia combinada en régimen diario, semanal o dos veces a la semana., ${ }^{2,8}$

La vía de administración es subcutánea y el régimen diario es la forma más efectiva. La dosis inicial usualmente es de $10 \mathrm{mg}$ al día y la dosis máxima de mantenimiento que se puede administrar es de $30 \mathrm{mg}$ al día. ${ }^{2}$ En casos específicos se puede administrar dos o una vez por semana. ${ }^{8}$ Se sugiere la medición de IGF-1 a las 4 a 6 semanas tras haber iniciado el tratamiento y luego de cada cambio de dosis hasta alcanzar el control bioquímico. Cuando se normalizan los valores de IGF1 , se debe monitorizar cada 3 a 6 meses. ${ }^{2}$

Entre los efectos adversos se incluyen: cambios en el tamaño del tumor, aumento de enzimas hepáticas y reacciones en el sitio de inyección. ${ }^{2,8,10}$ Las pruebas de función hepática se deben realizar una vez al mes los primeros 6 meses, de forma trimestral los siguientes 6 meses y luego bianualmente. Debido al posible crecimiento del tumor, se sugiere monitorizar de forma seriada con resonancia magnética de la hipófisis. ${ }^{8} \mathrm{Sin}$ embargo, la progresión del tumor es rara; en el German Pegvisomant Observational Study, en los pacientes tratados con Pegvisomant, tras evaluaciones centradas en los estudios de imagen, se reportó entre un 2 y $3 \%$ de los sujetos, y no excedió la tasa esperada para pacientes con acromegalia no tratados con dicho fármaco. ${ }^{22}$

\section{Radioterapia}

La radioterapia se ha utilizado durante muchos años para el tratamiento de la acromegalia, usualmente como una terapia adyuvante tras falla en la cirugía hipofisaria (tumor residual) o si la terapia médica falla o no es tolerada. ${ }^{6,8,10,23}$ La radioterapia tiene efectos variables en la reducción de los niveles de GH e IGF-1 y la respuesta se puede retrasar incluso 10 años. Por esta razón usualmente reciben terapia médica durante este tiempo, hasta que la radioterapia alcance su efecto completo. ${ }^{6}$ Las concentraciones de IGF-1 disminuyen de forma más lenta que las de GH. Varios estudios han demostrado una tasa de remisión de $40-70 \%$ tras 10 años de tratamiento. ${ }^{23}$

Cuando se encuentra disponible, se recomienda la radiocirugía estereotáctica (que incluye bisturí de rayos gamma, CyberKnife, acelerador lineal o haz de protones) sobre la radioterapia convencional, a menos que exista gran cantidad de tumor residual o que el tumor esté próximo al quiasma óptico. ${ }^{6}$ La principal ventaja de la radiocirugía es que reduce la dosis de radiación recibida por tejido que se encuentra cerca del blanco porque los haces convergen en el objetivo. ${ }^{24}$

Entre las desventajas de la radioterapia se encuentran el retraso en el logro de la remisión bioquímica y el posible desarrollo de deficiencias hormonales a nivel hipofisario (hipopituitarismo). ${ }^{8,23}$ Por este motivo se debe dar seguimiento a la función adrenal, tiroidea y gonadal, al menos una vez al año. ${ }^{6,8}$ También se presenta el riesgo de tumores secundarios inducidos por radiación y radionecrosis. ${ }^{6}$

En caso de enfermedad recurrente, la irradiación por segunda vez puede ser aceptable como manejo después de un periodo de 3 a 4 años de la radioterapia previa a nivel hipofisario. Sin embargo, aumenta el riesgo de efectos acumulados por la radiación. ${ }^{6}$

\section{CONCLUSIONES}

La acromegalia, a pesar de ser una enfermedad poco común, es importante tenerla presente debido a que un diagnóstico y tratamiento oportuno puede prevenir la morbilidad y mortalidad. Existen múltiples opciones para el tratamiento de esta patología, siendo el tratamiento quirúrgico el de elección, sin embargo se debe individualizar el manejo y es deseable que la decisión sea tomada por un equipo multidisciplinario.

\section{BIBLIOGRAFÍA}

1. De Herder WW. The History of 
Acromegaly. Neuroendocrinology. 2016;103(1):7-17

2. Giustina A et al. Use of Pegvisomant in acromegaly. An Italian Society of Endocrinology guideline. J Endocrinol Invest 2014;37:1017-1030

3. Eugster E et al. Gigantism. Endotext. (2015). Disponible en: http://www.ncbi.nlm.nih.gov/pubmed/25905378

4. Cordido F García JA Marazuela M Torres E. Guía práctica de diagnóstico y tratamiento de la acromegalia. Endocrinol Nutr. 2013;60(8):457.e1457.e15

5. Lugo G Pena L Cordido F. Clinical Manifestations and Diagnosis of Acromegaly. International Journal of Endocrinology 2012;(1):540398

6. Silverstein J. Need for improved monitoring in patients with acromegaly. Endocrine Connections 2015;4, R59-R67.

7. Dineen R Stewart P Sherlock M. Acromegaly- Diagnosis and Clinical Management. 2016. Publicado por Oxford University Press a favor de Association of Physicians Disponible en: $\quad$ DOI: http://dx.doi.org/10.1093/qjmed/hcw004 hew004

8. American Association of Clinical Endocrinologists Medical Guidelines for Clinical Practice for the Diagnosis and Treatment of Acromegaly- 2011 update. Endocr Pract. 2011;17(Suppl 4) 3

9. Melmed S et al. A consensus on the diagnosis and treatment of acromegaly complications. Pituitary 2013;16:294302

10. The Endocrine Society. Acromegaly: An Endocrine Society Clinical Practice Guideline. J Clin Endocrinol Metab, November 2014;99(11):3933-3395
11. Rúa C Latorre G Campuzano G. Diagnóstico de acromegalia. Medicina y Laboratorio 2011;17:511-531

12. Berlanga E. Diagnóstico bioquímico del exceso de secreción de somatotropina (I). Endocrinol Nutr. 2006;53(9):559-564

13. Chanson P Salenave S. Acromegaly. Orphanet Journal of Rare Diseases 2008; 3:17

14. Hernández S Valverde $\mathrm{O}$ Esquivel MA. Remisión bioquímica de pacientes acromegálicos sometidos a cirugía endoscópica. Revista Médica de la Universidad de Costa Rica 2014; $8(2): 44-52$

15. Pereira OL Palay MS Rodríguez A. Acromegalia: diagnóstico y tratamiento. MEDISAN 2015;19(3):403

16. Giustina $\mathrm{P}$ et al. A Consensus on Criteria for Cure of Acromegaly. J Clin Endocrinol Metab. 2010;95(7):31413148

17. Barkan A et al. Management of acromegaly in Latin America: expert panel recommendations. Pituitary 2010; 13:168-175

18. Ronchi CL et al. Long-term basal and dynamic evaluation of hypothalamic-pituitary-adrenal (HPA) axis in acromegalic patients. Clinical Endocrinology 2008;69: 608-612

19. Giustina A et al. A consensus on the medical treatment of acromegaly. Nat. Rev. Endocrinol. 2014;10:243-248

20. Plöckinger U. Medical Therapy of Acromegaly. International Journal of Endocrinology Volume 2012, Article ID 268957, doi:10.1155/2012/268957

21. Samson S. Pasireotide in Acromegaly: An Overview of Current Mechanistic and Clinical Data. Neu- 
roendocrinology 2015;102:8-17

22. Buchfelder $\mathrm{M}$ et al. Pituitary tumor size in acromegaly during pegvisomant treatment: experience from MR re-evaluations of the German Pegvisomant Observational Study. European Journal of Endocrinology 2009;161;27-35

23. González B et al. Efficacy and Safety of Radiotherapy in Acromegaly. Archives of Medical Research 2011;42: 48-52

24. Rowland NC Aghi MK. Radiation treatment strategies for acromegaly. Neurosurg Focus 2010;29 (4):E12

\section{CONFLICTO DE INTERÉS Y/O} AGRADECIMIENTOS

Las autoras declaran que no existió ningún conflicto de interés en el presente reporte. 